\title{
COGNITIVE ASSESSMENT IN THE DIMENSIONAL APPROACH OF SCHIZOPHRENIA SPECTRUM DISORDERS USING CRDPSS: IS COGNITIVE DECLINE A POSSIBLE OBJECTIVE CLINICAL BIOMARKER?
}

\section{P. A. Malliaris ${ }^{1,2}$, K. Bonotis ${ }^{1}$ \\ ${ }^{1}$ University Hospital of Larisa, Department of Psychiatry, Faculty of Medicine, University of Thessaly, \\ ${ }^{2}$ General Hospital of Karditsa}

\section{Introduction:}

Schizophrenia spectrum disorders are characterized by heterogeneity (1), which indicates that, until research will allow a more pathogenetic diagnostic differentiation (2), a dimensional approach may arise as a useful alternative in clinical practice. The objective of this research is to evaluate the clinical value of the dimensional approach, which allows the independent, as well as the multi-factorial correlation of the dimensions of psychotic symptoms with other variables, focusing mostly in the cognitive decline observed in patients (3).

\section{Background:}

DSM-V, in addition to the categorical model of diagnosis (4), proposes an 8-item measure, CRDPSS (Clinician-Rated Dimensions of Psychosis Symptom Severity), which assesses the severity of eight important symptoms in psychotic disorders, such as delusions, hallucinations, disorganized speech, abnormal psychomotor behavior, negative symptoms, impaired cognition, depression, and mania, on a 5point (0-4) scale. In this ordinal scale, 0 means absence of symptom, 1 that the symptom is equivocal and 2-4 that the symptom is present, classified according to severity as mild-2, moderate-3 or severe-4. This measure is designed, among other goals, in order to capture meaningful variations in the severity of symptoms, used in research on pathophysiological mechanisms (5).

\section{Material and Methods:}

65 Patients diagnosed with schizophrenia spectrum disorders, attended in the recently founded Outpatient Department of Early Intervention in Psychosis of University of Thessaly, Greece and its affiliated psychiatric clinics, were evaluated the last 18 months, using the CRDPSS measure. The validated Greek version of the MoCA (Montreal Cognitive Assessment) test, consisting of 30 cognitive tasks, was used for the classification of cognitive impairment (6).

\section{Results:}

Cognitive status, as assessed with MoCA scale, is inversely correlated with the severity of most (delusions $\mathrm{p}=0,003$, disorganized speech $\mathrm{p}=0,008$, abnormal psychomotor behavior $\mathrm{p}=0.042$, mania $\mathrm{p}<0,001$ ) but not all dimensions of psychosis symptoms.

CRDPSS (Clinician-Rated Dimensions of Psychosis Symptom Severity)

\begin{tabular}{|r|c|c|c|c|c|c|}
\hline \multicolumn{1}{|c|}{ Domain } & 0 & 1 & 2 & 3 & 4 & Score \\
\hline I. Hallucinations & Not present & Equivocal & Present, but mild & Present and moderate & Present and severe & \\
\hline $\begin{array}{r}\text { II. Delusions } \\
\text { III. Disorganized } \\
\text { speech }\end{array}$ & Not present & Equivocal & Present, but mild & Present and moderate & Present and severe & \\
\hline $\begin{array}{r}\text { IV. Abnormal } \\
\text { psychomotor behavior }\end{array}$ & Not present & Equivocal & Present, but mild & Present and moderate & Present and severe & \\
\hline $\begin{array}{r}\text { V. Negative Symptoms } \\
\text { VI. Impaired Cognition }\end{array}$ & Not present & Equivocal & Present, but mild & Present and moderate & Present and severe & \\
\hline VII. Depression & Not present & Equivocal & Present, but mild & Present and moderate & Present and severe & \\
\hline VIII. Mania & Not present & Equivocal & Present, but mild & Present and moderate & Present and severe & Prend severe \\
\hline
\end{tabular}

We propose the use of CRDPSS7 index which consists of the 5 symptoms used in the categorical diagnosis of schizophrenia, plus the affective domains, as the sum of severity of all symptoms. This proposed index is also inversely correlated with cognitive status, as assessed with MoCA scale $(\mathrm{p}=0,004)$. Nevertheless, the abovementioned index should not be used as an alternative to the dimensional approach that DSM V suggests with the use of CRDPSS.

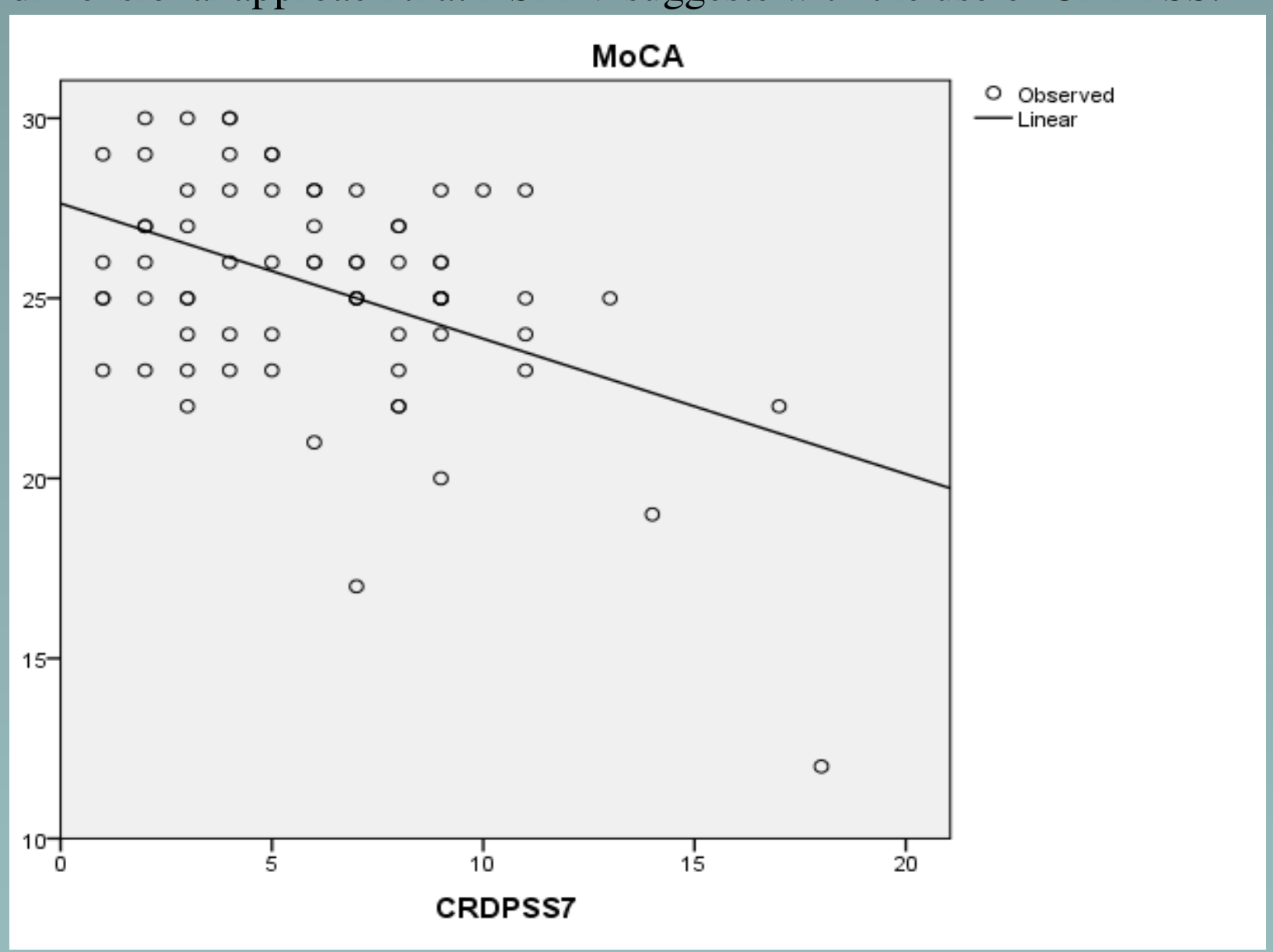

Absence of symptoms (0) and equivocal symptoms (1) were not distinguished in any of the domains, suggesting that the scale may not be quite useful for early intervention patients with prodromal symptoms. There were no differences considering sex, age, medication (number, type, way of distribution), and diagnosis (with the exception of organic psychosis, $\mathrm{p}<0,001$ ), while university education status was only distinguished from elementary education status $(\mathrm{p}=0,002)$. These results may suggest that adaptation for these factors may not be needed The most frequently wrong answered tasks was fluency (55\%), repetition $(31 \%)$, recall $(29 \%)$, and visuospatial functions $(22 \%)$.

\section{Conclusions:}

We propose the consistent cognitive assessment of all patients diagnosed with schizophrenia spectrum disorders, since cognitive status is inversely correlated with the severity of psychotic symptoms. Future studies should further examine the use of recent measured cognitive decline as a possible objective clinical sign of relapse and the implementation of new cognitive tests, especially for such patients.

Van Rheenen TE, Lewandowski KE Tan EJ, Ospina LH, Ongur D, Neill E, Gurvich C, Pantelis C, Malhotra AK, Rossell SL, Burdick KE, Characterizing cognitive heterogeneity on the schizophrenia-bipolar disorder spectrum. Psychol Med. 2017 Jul;47(10):1848-1864. doi: 10.1017/S0033291717000307

Strik W, Stegmayer K, Walther S, Dierks T, Systems Neuroscience of Psychosis: Mapping Schizophrenia Symptoms onto Brain Systems. Neuropsychobiology. 2017;75(3):100-116. doi: 10.1159/000485221. Oya K, Okahisa Y, Takaki M, Hashimoto N, Kato M, Onitsuka T, Ueno T, Ohnuma T7, Kasai K, Ozaki N, Sumiyoshi T, Imura O, Hashimoto R; for COCORO. Estimated cognitive decline in patients with schizophrenia: A multicenter study. Psychiatry Clin Neurosci2017May:71(5):294-300.doi: $0.1111 /$ pcn.12474.
. Mattila T, Koeter M, Wohlfarth T, Storosum J, van den Brink W, de Haan L, Derks E, Leufkens H, Denys D, Impact of DSM- 5 changes on the diagnosis and acute treatment of schizophrenia, Schizophr Bull. 2015 May;41(3):637and acute treatment of schizophen

American Psychiatric Association. (2013). Diagnostic and statistical manual American Psychiatric Associa of mental disorders (5th ed). Konstonoulos K, Vogazianos P, Doskas T. Normative Data of the Montreal Cognitive Assessment in the Greek Population and Parkinsonian Dementia. Arch Clin Neuro-psychol. 2016 May;31(3):246-53. doi: 10.1093/arclin/acw002 